\title{
Corrigendum: Structure of EF-G-ribosome complex in a pretranslocation state
}

Yun Chen, Shu Feng, Veerendra Kumar, Rya Ero \& Yong-Gui Gao

Nat. Struct. Mol. Biol. 20, 1077-1084 (2013); published online 4 August 2013; corrected after print 12 January 2015

In view of the differences between our findings reported in this study and in Tourigny et al. (Science 340, 1235490, 2013), we have rerefined the structure with Refmac (Murshudov, G.N. et al., Acta Crystallogr. D Biol. Crystallogr. 67, 355-367, 2011). This resulted in better statistics and revealed that the structure contained tRNA ${ }^{\text {Phe }}$ in the P/E site, as in Tourigny et al., and not tRNA ${ }^{\mathrm{fMet}}$ as we had originally interpreted. (Both tRNA ${ }^{\mathrm{fMet}}$ and tRNA $^{\text {Phe }}$ had been included for crystallization.) Thus, the structure reported here is essentially identical to that reported in Tourigny et al., and we thank D. S. Tourigny, G. Murshudov and V. Ramakrishnan for alerting us about this issue after they had rerefined our data with Refmac. We have updated the coordinates accordingly (PDB 4CR1) and made the following corrections: (i) on pages 1078 and page 1080, tRNA ${ }^{\mathrm{fMet}}$ was changed to tRNA ${ }^{\text {Phe; }}$ (ii) on page 1081, including Figure 4b, A43 was changed to C43, and A43-U27 was changed to C43-G27; (iii) on page 1082, G42 was changed to C42. The text and Figure 4 have been updated to reflect the revised information as of 12 January 2015.

\section{Corrigendum: Cross-talking noncoding RNAs contribute to cell-specific neurodegeneration in SCA7}

Jennifer Y Tan, Keith W Vance, Miguel A Varela, Tamara Sirey, Lauren M Watson, Helen J Curtis, Martina Marinello, Sandro Alves, Bruno R Steinkraus, Sarah Cooper, Tatyana Nesterova, Neil Brockdorff, Tudor A Fulga, Alexis Brice, Annie Sittler, Peter L Oliver, Matthew J Wood, Chris P Ponting \& Ana C Marques Nat. Struct. Mol. Biol. 21, 955-961 (2014); published online 12 October 2014; corrected after print 18 February 2015

In the version of this article initially published, Supplementary Figure 4k showed levels of mature miRNA-124 instead of miR-124 precursor. The error has been corrected in the Supplementary Text and Figures file and in the HTML and PDF versions of the article. 